\title{
Discovery of starspots on Vega ${ }^{\star}$
}

\section{First spectroscopic detection of surface structures on a normal A-type star}

\author{
T. Böhm ${ }^{1,2}$, M. Holschneider ${ }^{3}$, F. Lignières ${ }^{1,2}$, P. Petit ${ }^{1,2}$, M. Rainer ${ }^{4}$, F. Paletou ${ }^{1,2}$, G. Wade $^{6}$, E. Alecian ${ }^{5}$, \\ H. Carfantan ${ }^{1,2}$, A. Blazère ${ }^{1,2}$, and G. M. Mirouh ${ }^{1,2}$ \\ 1 Université de Toulouse, UPS-OMP, IRAP, Toulouse, France \\ e-mail: torsten . boehm@irap. omp. eu \\ 2 CNRS; IRAP; 14 avenue Édouard Belin, 31400 Toulouse, France \\ 3 Institut für Mathematik, Universität Potsdam, DYCOS, 14469 Potsdam, Germany \\ 4 INAF-Osservatorio Astronomico di Brera, via E. Bianchi 46, 23807 Merate, Italy \\ 5 Observatoire de Grenoble, Université Joseph Fourier, IPAG, 38041 Grenoble, France \\ ${ }^{6}$ Dept. of Physics, Royal Military College of Canada, PO Box 17000, Stn Forces, Kingston, Canada K7KK 7B4
}

Received 27 November 2014 / Accepted 20 March 2015

\section{ABSTRACT}

\begin{abstract}
Context. The theoretically studied impact of rapid rotation on stellar evolution needs to be compared with these results of highresolution spectroscopy-velocimetry observations. Early-type stars present a perfect laboratory for these studies. The prototype A0 star Vega has been extensively monitored in recent years in spectropolarimetry. A weak surface magnetic field was detected, implying that there might be a (still undetected) structured surface. First indications of the presence of small amplitude stellar radial velocity variations have been reported recently, but the confirmation and in-depth study with the highly stabilized spectrograph SOPHIE/OHP was required.

Aims. The goal of this article is to present a thorough analysis of the line profile variations and associated estimators in the early-type standard star Vega (A0) in order to reveal potential activity tracers, exoplanet companions, and stellar oscillations.

Methods. Vega was monitored in quasi-continuous high-resolution echelle spectroscopy with the highly stabilized velocimeter SOPHIE/OHP. A total of 2588 high signal-to-noise spectra was obtained during $34.7 \mathrm{~h}$ on five nights (2 to 6 of August 2012) in high-resolution mode at $R=75000$ and covering the visible domain from 3895-6270 ̊. For each reduced spectrum, least square deconvolved equivalent photospheric profiles were calculated with a $T_{\text {eff }}=9500$ and $\log g=4.0$ spectral line mask. Several methods were applied to study the dynamic behaviour of the profile variations (evolution of radial velocity, bisectors, vspan, 2D profiles, amongst others).

Results. We present the discovery of a spotted stellar surface on an A-type standard star (Vega) with very faint spot amplitudes $\Delta F / F c \sim 5 \times 10^{-4}$. A rotational modulation of spectral lines with a period of rotation $P=0.68 \mathrm{~d}$ has clearly been exhibited, unambiguously confirming the results of previous spectropolarimetric studies. Most of these brightness inhomogeneities seem to be located in lower equatorial latitudes. Either a very thin convective layer can be responsible for magnetic field generation at small amplitudes, or a new mechanism has to be invoked to explain the existence of activity tracing starspots. At this stage it is difficult to disentangle a rotational from a stellar pulsational origin for the existing higher frequency periodic variations.

Conclusions. This first strong evidence that standard A-type stars can show surface structures opens a new field of research and ask about a potential link with the recently discovered weak magnetic field discoveries in this category of stars.
\end{abstract}

Key words. starspots - stars: early-type - stars: rotation - stars: oscillations - stars: individual: Vega - asteroseismology

\section{Introduction}

The role rapid rotation plays on the stellar interior and its evolution represents a very challenging research topic as of today. Rapidly rotating stars reveal many unanswered questions in the domain of observations, theory, and modelling. The only known way to study stellar interiors is through asteroseismology and its associated observational techniques, which are based either on photometry or high-resolution spectroscopy. In addition, the detection and observation of activity tracing structured stellar surfaces can also contribute significant constraints on stellar evolution models.

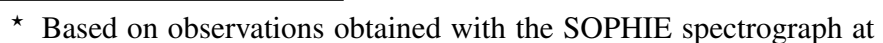
the $2 \mathrm{~m}$ OHP telescope operated by the Institut National des Sciences de l'Univers (INSU) of the Centre National de la Recherche Scientifique of France (CNRS).
The recent detection of a very weak magnetic field in Vega was reported in Lignières et al. (2009). The $-0.6 \pm 0.3 \mathrm{G}$ diskaveraged line-of-sight component of the surface magnetic field can reach peak values of $7 \mathrm{G}$ (Petit et al. 2014). Lignières et al. (2009) and later Petit et al. (2010) have concluded that a previously unknown type of magnetic star exists in the intermediatemass domain and that Vega may well be the first confirmed member of a much larger, as yet unexplored, class of weaklymagnetic stars. The Zeeman-Doppler imaging of the magnetic field topology (Petit et al. 2010, 2014) shows that apart from a prominent polar magnetic region, a few other magnetic spots are reconstructed at lower latitude. Petit et al. (2010) conclude that an important help for distinguishing between a potential fossil or dynamo origin of the magnetic field would be to investigate the long-term stability of the observed field geometry, because 
a dynamo-generated field is likely to show some temporal variability. Detecting surface structures in unpolarized light would largely contribute to a better understanding of the origin of magnetic fields in these stars and of the role the rotation could play.

Large scale surveys of A-type stars with the Kepler satellite revealed low frequency periodic variations in $28 \%$ of the sample interpreted as linked to the stellar rotation frequency and associated rotational modulation (Balona 2011). The author suggests that the light variations in A-type stars may possibly be due to starspots or other corotating structures and that A-star atmospheres may not be quiescent as previously supposed. In a more recent article on Kepler data, Balona (2013) reports that photometric indications for the period of rotation are detected in 875 A-type stars (40\%). From the amplitude distribution, he concludes that the sizes of starspots in A-type stars are similar to the largest sunspots. He concludes that A-type stars are active and, like cooler stars, have starspots and flares. Providing direct detection of starspotted surfaces in A-type stars is therefore a necessary next step for confirming this thesis.

The A0 photometric standard star Vega is a rapid rotator. Vega is seen pole-on with an inclination angle of $i=5-7^{\circ}$, a result ascertained by photometric (Gray 1985), spectroscopic (Gulliver et al. 1994), and interferometric (Aufdenberg et al. 2006; Peterson et al. 2006; Monnier et al. 2012) observations. Vega's $v \sin i$ has been determined by Hill et al. (2004): $21.9 \pm 0.1 \mathrm{~km} \mathrm{~s}^{-1}$. A new determination by Yoon et al. (2010) concludes on slightly lower values, depending on the model: $20.48 \pm 0.11 \mathrm{~km} \mathrm{~s}^{-1}$ or $20.80 \pm 0.11 \mathrm{~km} \mathrm{~s}^{-1}$.

While spectroscopic analysis and modelling by Takeda et al. (2008) and Hill et al. (2010) differ slightly in equatorial velocities $\left(\sim 175 \mathrm{~km} \mathrm{~s}^{-1}\right.$ versus $211 \pm 4 \mathrm{~km} \mathrm{~s}^{-1}$, respectively), first interferometric studies by Peterson et al. (2006) and Aufdenberg et al. (2006) led to finding a much higher equatorial velocity of $\sim 275 \mathrm{~km} \mathrm{~s}^{-1}$.

Petit et al. (2010) have announced that the short-term evolution of polarized signatures in Vega is consistent with a rotational period of $0.732 \pm 0.008 \mathrm{~d}$, where the error bar has been underestimated (priv. comm.). Alina et al. (2012) worked on the NARVAL/TBL 2010 data set (Alina et al. 2012; Böhm et al. 2012) and detected a period of $0.678 \pm_{0.029}^{0.036} \mathrm{~d}$. Budkovskaya (2013) analysed the results of 1312 longitudinal magnetic field measurements obtained during $15 \mathrm{yr}$ of observations performed at the Crimean Astrophysical Observatory and propose that the magnetic field variations are caused by stellar rotation; their derived stellar rotation period corresponds to 0.6225503 days. Both determined periods are significantly longer than values expected from earlier interferometry-based models, but compatible with the results of spectroscopic modelling of the gravitydarkened photospheric lines in Vega (Takeda et al. 2008). Based on new interferometric CHARA/MIRC observations, Monnier et al. (2012) conclude that a more slowly rotating model was compatible with the new interferometric data, thereby reconciling these new results with those determined by spectroscopy.

As reviewed by Gray (2007), Vega has been used for more than $150 \mathrm{yr}$ as a photometric and spectrophotometric standard. Still, very low photometric variability has occasionally been reported at the $1-2 \%$ level. Hill et al. (2010) report low-level variations in Ti II 4529 AA profiles on the time basis of several years. First evidence of pulsations in Vega was suspected in three data sets (3 nights NARVAL/TBL 2008 - 3 nights ESPADONS/CFHT 2009 - 5 nights TBL 2010) corresponding to a total of 4478 quasi-continuous, high-resolution $(R>65000)$ echelle spectra Böhm et al. (2012). Least square deconvolved
(LSD) profiles (Donati et al. 1997) were obtained for each spectrum, representing the photospheric absorption profile potentially deformed by the presence of pulsations, and telluric lines were used as a velocity reference. All three data sets revealed to be residual periodic variations with the following frequencies and amplitudes: 5.32 and $9.19 \mathrm{~d}^{-1}\left(A \approx 6 \mathrm{~m} \mathrm{~s}^{-1}\right)$ in $2008,12.71$ and $13.25 \mathrm{~d}^{-1}\left(A \approx 8 \mathrm{~m} \mathrm{~s}^{-1}\right)$ in 2009 , and 5.42 and $10.82 \mathrm{~d}^{-1}$ $\left(A \approx 3-4 \mathrm{~m} \mathrm{~s}^{-1}\right)$ in 2010 . However, owing to a lack of intrinsic stability of the employed spectropolarimeters, it was too early to conclude that the variations were due to stellar pulsations, so it was concluded that their confirmation with a highly stable spectrograph was the necessary next step. The results of a five-night survey with the highly stabilized spectrograph SOPHIE/OHP is presented in this article.

Section 2 describes the observations and data reduction; Sect. 3 presents the results concerning rotation and surface spots, potential exoplanet companions, and stellar oscillations and provides the discussion of the results; and Sect. 4 gives the conclusion of the article.

\section{Observations and data reduction}

In August 2012, 2588 high-resolution $(R=75000)$ spectra of Vega were acquired during $34.7 \mathrm{~h}$ on five consecutive nights, using the ultrastable echelle spectrograph SOPHIE/OHP. The log of observations is reported in Table 1.

The typical signal-to-noise ratio $(\mathrm{S} / \mathrm{N})$ per resolved element was around 800 at $520 \mathrm{~nm}$. Spectra with low $\mathrm{S} / \mathrm{N}(S / N<300$ per resolved element) were rejected. Because of the excellent intrinsic stability of the spectrograph (of the order of $2-3 \mathrm{~m} \mathrm{~s}^{-1}$ using simultaneous $\mathrm{Th} / \mathrm{Ar}$ calibration), no long-term trends related to instrumental shifts were present in the data set. The data reduction procedure was reduced to its minimum based on the standard SOPHIE data reduction pipeline, in order to avoid introducing any artefacts.

We then calculated photospheric LSD-profiles for all stellar spectra (Donati et al. 1997) using the original well-established prescription and the original code. The resulting equivalent photospheric profiles have been used in many domains, such as magnetic structures (in polarimetry), ZDI (Zeeman-Doppler Imaging), asteroseismology, Doppler imaging, etc. The article by Reiners \& Schmitt (2003) demonstrates that LSD does not modify the original shape of line profiles. Recently, Kochukhov et al. (2010) have analysed the behaviour of LSD in depth, mostly concerning the polarimetric aspects. LSD applied to intensity spectra corresponds to a (weighted) cross-correlation technique. This implies that all conclusions obtained concerning the validity of cross-correlation techniques applied to Doppler searches for exoplanets also apply to LSD. This includes radial velocities and bisectors. The regime in which LSD is used in this article concerns fluctuations of several $\mathrm{m} / \mathrm{s}$, which implies that our study is located very comfortably within the application regime of cross-correlation techniques, as demonstrated by many publications on exoplanets see for example the capacity of these methods on articles concerning HARPS observations on $\alpha$ Cen by Dumusque et al. (2012) or the article by Bazot et al. (Bazot et al. 2011) on 18 Sco. In polarimetry, LSD has proved to be extremely sensitive to signal variations of the order of $10^{-5}$ of the continuum (Aurière et al. 2009, 2010; Lignières et al. 2009). LSD conserves the asymmetries of the spectral lines and therefore also of the bisectors, as demonstrated by articles on cool stars (e.g. articles on $\xi$ Boo: Petit et al. 2005; Morgenthaler et al. 2012). In summary, LSD profiles have been used successfully to study line profile variations in many asteroseismological articles 
Table 1. Log of the spectroscopic observations of Vega.

\begin{tabular}{ccccccl}
\hline \hline $\begin{array}{c}\text { Date } \\
(1)\end{array}$ & $\begin{array}{c}\text { BJD }_{\text {first }} \\
(2)\end{array}$ & $\begin{array}{c}\mathrm{BJD}_{\text {last }} \\
(3)\end{array}$ & $\begin{array}{c}N_{\text {spec }} \\
(4)\end{array}$ & $\begin{array}{c}t_{\text {exp }}(\mathrm{s}) \\
(5)\end{array}$ & $\begin{array}{c}t_{\text {cov }}(\mathrm{h}) \\
(6)\end{array}$ & $\begin{array}{l}S / N \\
(7)\end{array}$ \\
\hline Aug. 2 2012 & 6142.3308 & 6142.6238 & 425 & $17-30$ & 7.0 & $884 \pm 202$ \\
Aug. 3 2012 & 6143.3528 & 6143.6436 & 629 & $10-15$ & 7.0 & $925 \pm 145$ \\
Aug. 4 2012 & 6144.3412 & 6144.6423 & 628 & $10-17$ & 7.2 & $850 \pm 121$ \\
Aug. 5 2012 & 6145.3788 & 6145.6442 & 402 & $13-17$ & 6.4 & $766 \pm 103$ \\
Aug. 6 2012 & 6146.3444 & 6146.6423 & 504 & $17-25$ & 7.1 & $808 \pm 115$ \\
\hline
\end{tabular}

Notes. (1) Date of the observation (UT). (2) and (3) Barycentric Julian date (mean observation, 2450 000+) of the first and the last stellar spectrum of the night, respectively; (4) number of high-resolution Vega spectra obtained; (5) exposure time (s); (6) total hours covered on the sky; (7) nightly average and standard deviation of $\mathrm{S} / \mathrm{N}$ per resolved element at $520 \mathrm{~nm}$.

(e.g. Uytterhoeven et al. 2008; Nardetto et al. 2014; Mantegazza et al. 2012; Antonello et al. 2006). This shows that the LSD technique is indeed suited to studying small line asymmetries.

To calculate the LSD profiles, we used a mask based on a Kurucz atmospheric model of spectral type A0, matching the fundamental parameters of Vega well: $T_{\text {eff }}=9500 \mathrm{~K}, \log g=$ 4.0 and solar metallicity. (It should be kept in mind that Vega is slightly underabundant.) The adopted effective temperature lies between the polar and equatorial temperatures as published in Takeda et al. (2008) and Hill et al. (2010), the latter concluding that there is a mean temperature of $9560 \pm 30 \mathrm{~K}$. As shown by Takeda et al. (2008), due to important gravitational darkening in this rapidly rotating star, weak lines show flat-bottomed profiles that are very different from the well-known rotationally broadened profiles observed in the stronger lines (see also Figs. 1 and 2 of Böhm et al. 2012). To avoid mixing very different photospheric line profile shapes, we selected only the stronger lines with an unbroadend depth between 0.3 and 1.0 and excluded hydrogen Balmer lines. We kept a subset of 299 lines covering the visible acquisition domain of SOPHIE/OHP from 389-627 nm. All lines with a photospheric shape were kept, without any selection of particular line parameters. A detailed analysis of the results related to different line lists reveals that there is a low impact of the selected rejection threshold $(0.1,0.2,0.3$, etc. $)$ on the detailed results of the different analysis presented in this study. However, the overall conclusions remain unaffected.

In a next step, we derived several quantities for each LSD profile. The bisector of the line was determined by calculating the exact position of the left- and right-hand sides of the profile for each depth (via interpolation of the local profile), with the bisector the average of these two positions as a function of profile depth. Vspan was calculated for each profile by measuring the difference between the upper and lower parts of the bisector (a kind of skewness); for this, we worked in relative profile height, the bottom of the profile set at 0 ., the continuum at 1 . Vspan was calculated as the difference between the medians of upper $[0.35,0.5]$ and lower $[0.1,0.25]$ bisector ranges. Ranges were defined by checking out different combinations in order to produce the clearest periodograms. Optimal ranges lie fairly low in the profile, which can be understood considering the nearly pole-on position of the star, since equatorial contributions are barely seen (those that cross the full line profile from $-v \sin i$ to $+v \sin i$ ). Figure 1 shows the corresponding mean rescaled LSD profile. The variation in vspan with time is shown in Figs. 2 and 3, the latter showing the low-frequency variations.

Radial velocity can be determined in several ways: crosscorrelation with an average profile, fitting of Gaussians or rotationally broadened profiles, first-moment determination and median determination of the lower part of the bisector, amongst others. Having computed the different methods on our data set,

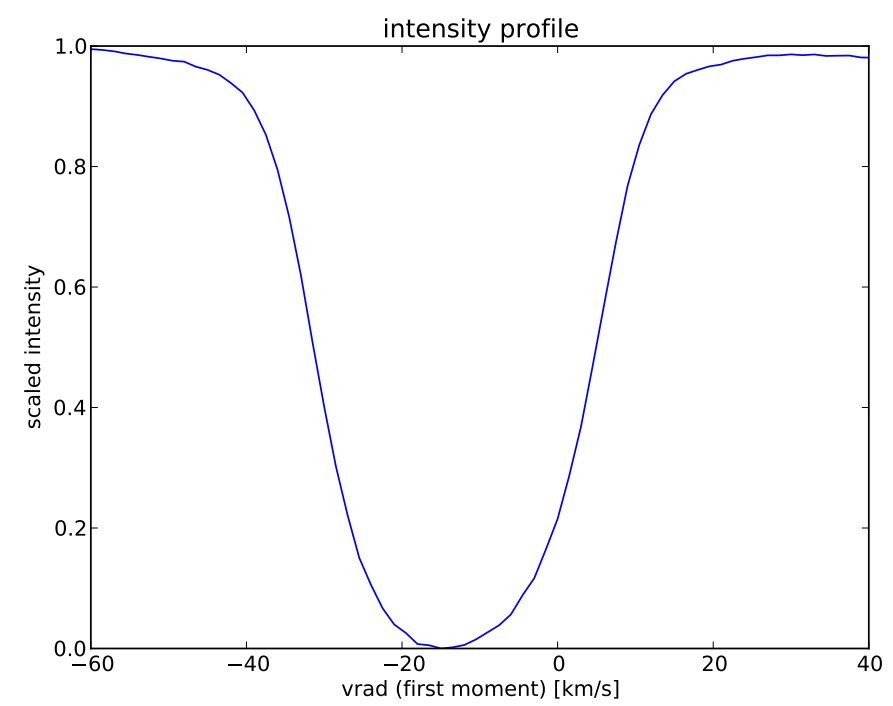

Fig. 1. Mean equivalent photospheric (LSD) profile of Vega, stronger lines alone, rescaled in depth.

we present the radial velocities determined by i) the first moment of the profile and ii) the median of the lower part of the line bisector range $[0.15,0.3]$, with the bottom of the line particularly sensitive to potential spot signatures crossing the line profile. The first moment has been calculated in the following way.

We let $I_{n}, n=1, \ldots N$, be a collection of intensities within a velocity bin with centre velocity $v_{n}$. The continuum level is at $I=1$. Since we consider absorption lines, we have $0 \leq I_{n} \leq 1$. The radial velocity estimate is then defined through

$\hat{v}_{r}=\frac{\sum_{n=1}^{N}\left(1-I_{n}\right) v_{n}}{\sum_{n=1}^{N}\left(1-I_{n}\right)}$.

In practice, the bins may be preselected based on some threshold $c$ such that $I_{n} \leq c$. In our case we used $c=0.5$ in order to be sensitive to the most variable parts of the LSD profile. The depth $c$ has some effect on the radial velocity determination, but the conclusions of this article remain valid as a whole. The variation of the radial velocity determined by the two different estimators (first moment and median of a lower bisector range) with time is shown in Fig. 4.

As can be seen in Tables 3 and 4, for a given frequency, the amplitude of the radial velocity variation strongly varies between the two estimators (first moment, bisector). This is not really surprising if considering that both determinations are significantly different and that a $10 \mathrm{~m} \mathrm{~s}^{-1}$ value corresponds to only $2 \times 10^{-4}$ of the overall width of the profile $\left(\simeq 2 v \sin i\right.$, i.e. $\left.44 \mathrm{~km} \mathrm{~s}^{-1}\right)$. 
SOPHIE/OHP 2012 observations
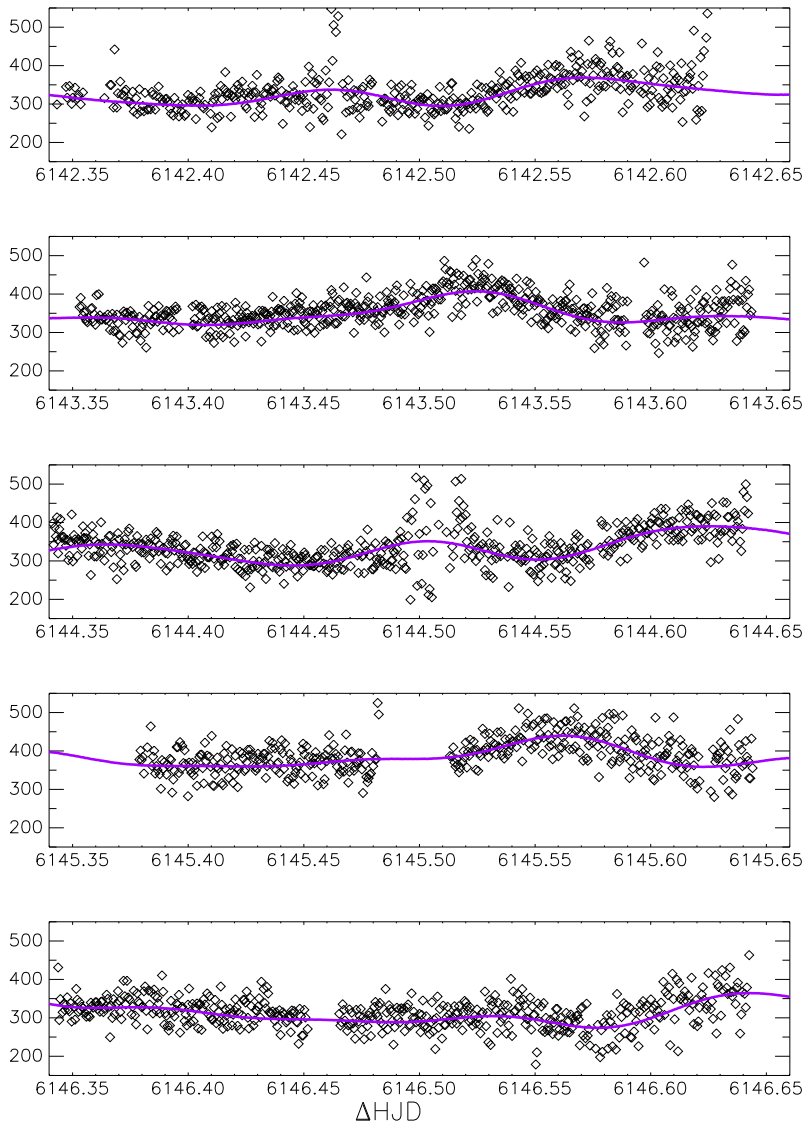

Fig. 2. Vspan variations of Vega (SOPHIE/OHP) in 2012. Each frame represents one night's observations. Superimposed (continuous line) is the result of the corresponding frequency analysis (Table 2). Time is expressed in $\mathrm{BJD}=2450000+\Delta \mathrm{BJD}$.

In a next step, we worked on the line profile variations themselves (as a function of velocity). The very small variations we discovered never exceeded $10^{-3}$ of the continuum. In traditional spectroscopic data sets, such small variations are generally not seen, mostly due to insufficiently precise data reduction or continuum normalization, amongst other issues. In our data set, continuum normalisation is performed several times. The overall spectra are normalized following reduction of the data, then the LSD procedure contains a local renormalisation, and eventually very tiny residuals (profile-to profile variations) in the continuum level are renormalized in the resulting LSD profiles. Clearly, the precision of normalization cannot be responsible for any of the observed variability. At these tiny levels of variation, particular care has to be taken to avoid any misinterpretation due to residual instrumental effects (see e.g. Sect. 3.2).

\section{Results and discussion}

\subsection{Periodicity analysis of vspan and radial velocity}

We started the search for periodicities in our line profile estimators by analysing the vspan data, which are insensitive to radial velocity calibration data (since they measure profile asymmetries to some extent, and no shifts). This asymmetry measurement turns out to be robust, and it provides similar frequency values, independently of the precise depth boundaries chosen.
Table 2. Frequencies and amplitudes of vspan variations measured on the LSD-profiles, and possible identifications.

\begin{tabular}{cccc}
\hline \hline ID & $\begin{array}{c}\text { Freq. } \\
\mathrm{d}^{-1}\end{array}$ & $\begin{array}{c}A \\
\mathrm{~m} \mathrm{~s}^{-1}\end{array}$ & Comment \\
\hline F1 & 1.457 & 69.32 & $F_{\text {rot }}$ \\
F2 & 2.02 & 22.93 & $2 F_{\text {rot }}-1$. \\
F3 & 0.40 & 47.52 & \\
F4 & 8.34 & 13.30 & $5 F_{\text {rot }}+1$. \\
F5 & 11.31 & 14.17 & $7 F_{\text {rot }}+1$. \\
F6 & 5.78 & 14.09 & $4 F_{\text {rot }}$ \\
F7 & 8.04 & 12.59 & \\
F8 & 16.21 & 8.23 & $11 F_{\text {rot }}$ \\
F9 & 5.43 & 9.05 & $3 F_{\text {rot }}+1$ \\
F10 & 10.70 & 8.92 & $9 F_{\text {rot }}-1$ \\
\hline
\end{tabular}

Notes. Only frequencies with a significance value above 4 are presented.

Table 3. Frequencies and amplitudes of vrad (bisector) variations measured on the LSD-profiles (median value of lower part 0.15-0.3).

\begin{tabular}{cccc}
\hline \hline ID & $\begin{array}{c}\text { Freq. } \\
\mathrm{d}^{-1}\end{array}$ & $\begin{array}{c}A \\
\mathrm{~m} \mathrm{~s}^{-1}\end{array}$ & Comment \\
\hline F1b & 1.44 & 20.20 & $F_{\text {rot }}$ \\
F2b -1 & 0.89 & 22.79 & $\mathrm{~F} 2 \mathrm{~b}=1.89$ \\
F3b & 9.34 & 13.91 & $7 F_{\text {rot }}-1 ? \mathrm{~F} 1_{2008} ?$ \\
F4b & 13.32 & 9.52 & $\mathrm{~F} 2_{2009}$ \\
F5b & 4.15 & 9.08 & \\
F6b & 5.71 & 11.46 & \\
F7b & 10.69 & 8.74 & $8 F_{\text {rot }}-1 ? \mathrm{~F}_{2009}-2 ? \mathrm{~F} 2_{2010} ?$ \\
F8b & 24.49 & 5.57 & $16 F_{\text {rot }}+1 ?$ \\
F9b & 9.94 & 6.17 & \\
\hline
\end{tabular}

Table 4. Frequencies and amplitudes of vrad (first moment) variations measured on the LSD profiles.

\begin{tabular}{cccc}
\hline \hline ID & $\begin{array}{c}\text { Freq. } \\
\mathrm{d}^{-1}\end{array}$ & $\begin{array}{c}A \\
\mathrm{~m} \mathrm{~s}^{-1}\end{array}$ & Comment \\
\hline F1c -1 & 0.77 & 6.16 & $\mathrm{~F} 1 \mathrm{c}=1.77$ \\
F2c & 3.34 & 4.32 & $3 F_{\text {rot }}-1 ? \mathrm{~F} 2_{2008}-2 ? \mathrm{~F} 1_{2010}-2 ?$ \\
F3c & 4.97 & 3.78 & $4 F_{\text {rot }}-1 ?$ \\
F4c & 1.48 & 4.01 & $F_{\text {rot }}$ \\
F5c & 24.47 & 2.17 & $16 F_{\text {rot }}+1 ?$ \\
F6c & 14.24 & 2.42 & $\mathrm{~F} 2_{2009}+1 ?$ \\
F7c & 8.29 & 2.12 & $5 F_{\text {rot }}+1 ?$ \\
F8c & 5.53 & 2.30 & \\
F9c & 11.78 & 2.61 & $\mathrm{~F} 1_{2009}-1 ? \mathrm{~F} 2_{2010}+1 ?$ \\
F10c & 14.67 & 2.68 & $10 F_{\text {rot }}$ \\
\hline
\end{tabular}

Notes. Additional frequencies with low amplitude $\left(<2.0 \mathrm{~m} \mathrm{~s}^{-1}\right)$ have been seen at 17.54, 20.15, 25.11, 30.44, and $54.92 \mathrm{~d}^{-1}$.

The Lomb Scargle periodogram of vspan is shown in Fig. 5. In a recent paper, Alina et al. (2012) had determined a $P_{\text {rot }}=$ $0.678 \pm_{0.029}^{0.036} \mathrm{~d}$ for Vega based on spectropolarimetric observations. We overplotted the corresponding rotational frequency of $1.47 \mathrm{~d}^{-1}$ at 0.3 height, as well as its harmonics (multiple of this frequency), the width of the bars corresponding to the reported error bars. The lower bars at 0.15 and 0.02 height indicate the position of the \pm 1 and 2-day aliases with respect to the rotational frequency comb, respectively.

An important result is that many detected periodicities in the range $0-15 \mathrm{~d}^{-1}$ correspond to the stellar rotation frequency (and its harmonics and aliases), and are in perfect agreement with the 


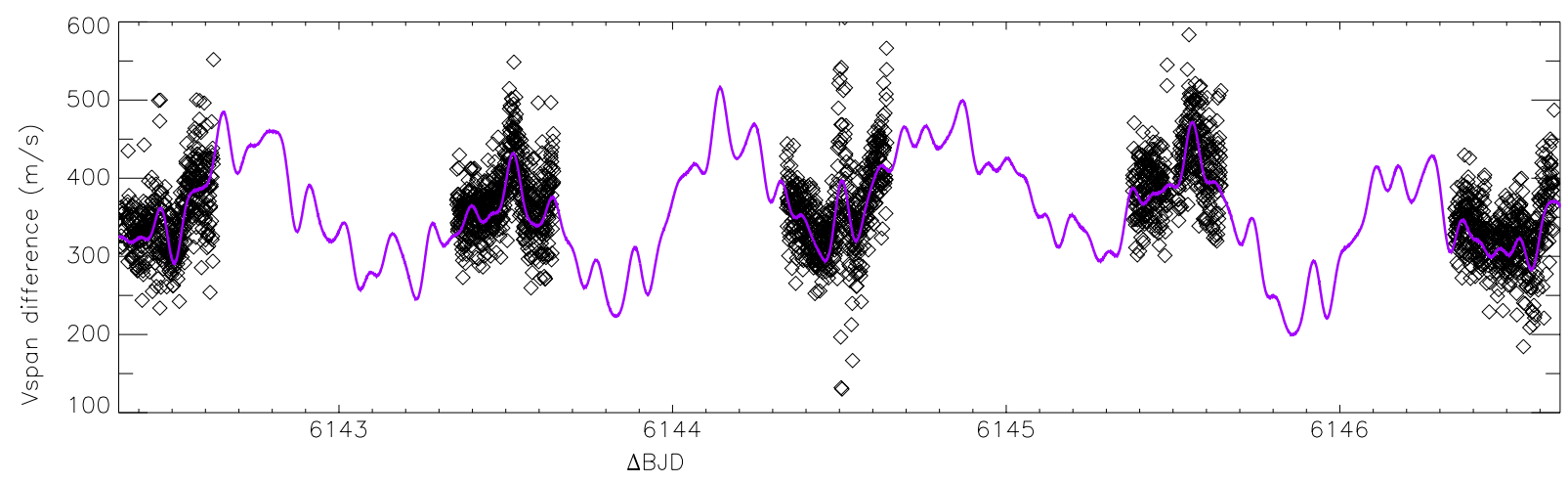

Fig. 3. Low-frequency vspan variations of Vega (SOPHIE/OHP 2012). Superimposed (continuous line) is the result of the corresponding frequency analysis as listed in Table 2. Time is expressed in $\mathrm{BJD}=2450000+\Delta \mathrm{BJD}$. The period of rotation can clearly be seen in this figure.

SOPHIE/OHP 2012 - radial velocity (bisector)

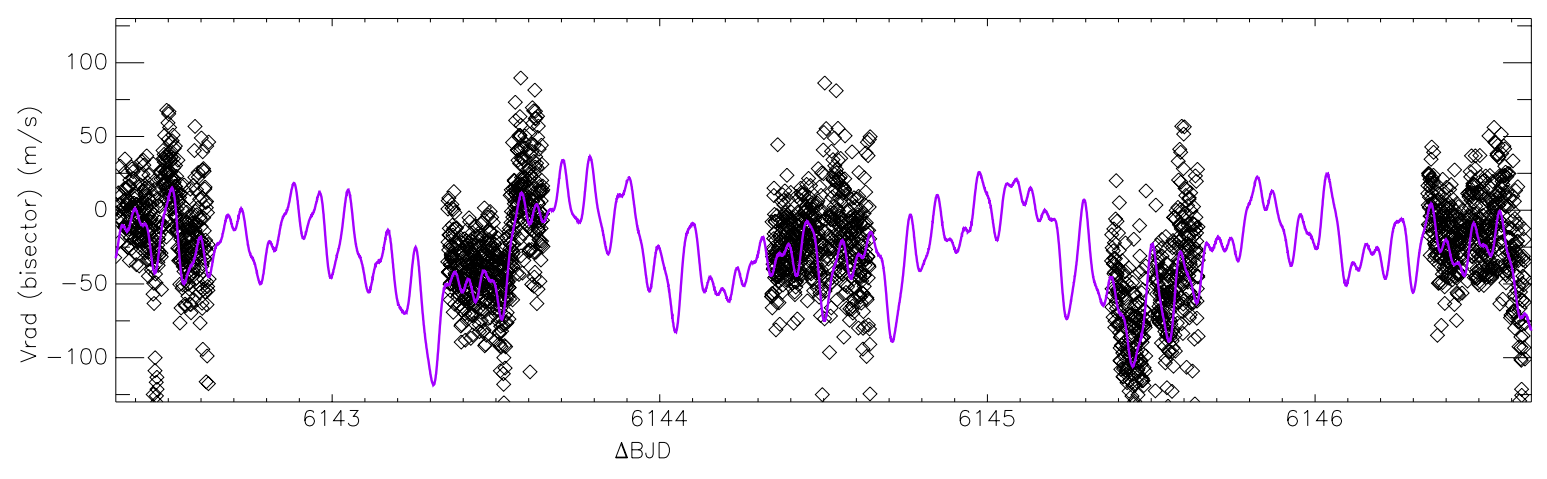

SOPHIE/OHP 2012 - radial velocity (first moment)

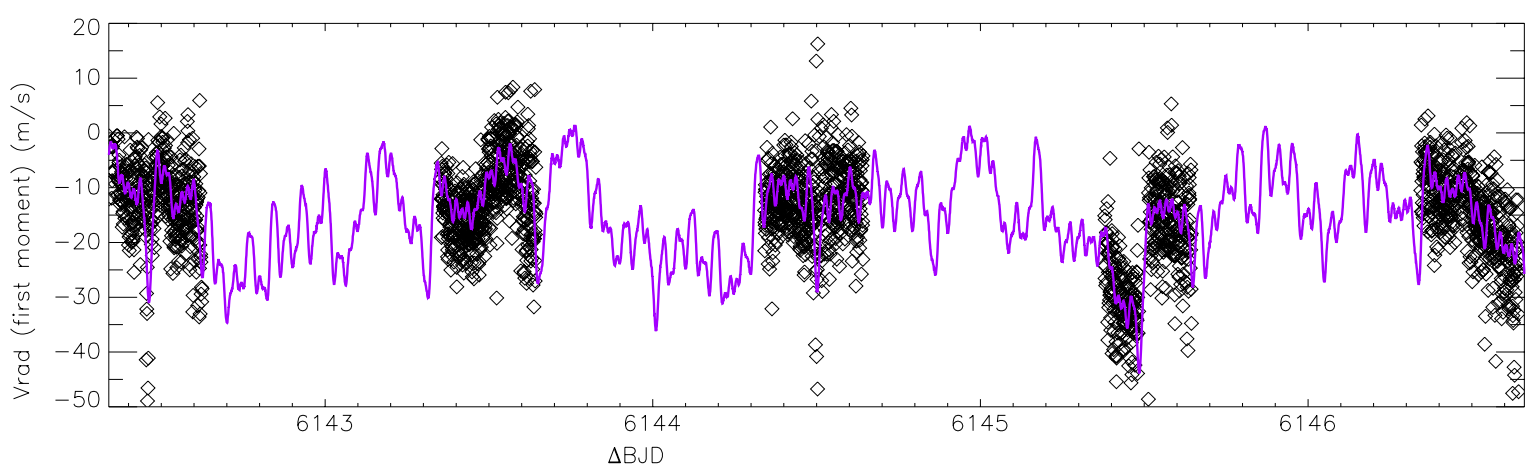

Fig. 4. Radial velocity variations of Vega (SOPHIE/OHP 2012) measured by the median of a lower bisector range (top) and first moment (bottom). Superimposed (continuous line) is the result of the corresponding frequency analysis as listed in Tables 3 and 4 . Time is expressed in BJD = $2450000+\Delta$ BJD. Both radial velocity determinations follow the same trend without being identical.

rotation period as published by Alina et al. (2012). This provided us with a first proof that surface structures should cross the photospheric equivalent line profile, leading to a rotational modulation of the vspan parameter.

Table 2 present the corresponding periodicity analysis of this vspan time series based on the SigSpec tool (Reegen 2007). SigSpec calculates the spectral significance of an amplitude A by $\operatorname{sig}(A)=-\log \left[\Phi_{\mathrm{FA}}(A)\right]$, where $\Phi_{\mathrm{FA}}$ indicates the false alarm probability. To do this, SigSpec computes the probability density function (pdf) of white noise in Fourier space. The integration of the pdf yields the false-alarm probability that white noise in the time domain produces an amplitude of at least A. The sig threshold for the determination of the prewhitening sequence was equal to four, which indicates that the considered amplitude level is due to noise in fewer than one out of $10^{4}$ cases. Only these frequencies have been listed in Tables 2-4. These frequencies are formally reliable; however, one must keep in mind that the measured entities can carry intrinsic errors and biases, which cannot easily be discarded before the subsequent frequency analysis.

Kurtz \& Müller (1999) estimate the uncertainty in the frequency to be approximately $1 /(4 \Delta T)$, where $\Delta T$ is the time span of the data set. In our case, this would yield an uncertainty of $0.05 \mathrm{~d}^{-1}$. The last column of the table proposes some identification with the rotation comb (only the star's rotation frequency $F_{\text {rot }}$, its harmonics, and $\pm 1 \mathrm{~d}$ window function aliases). For 

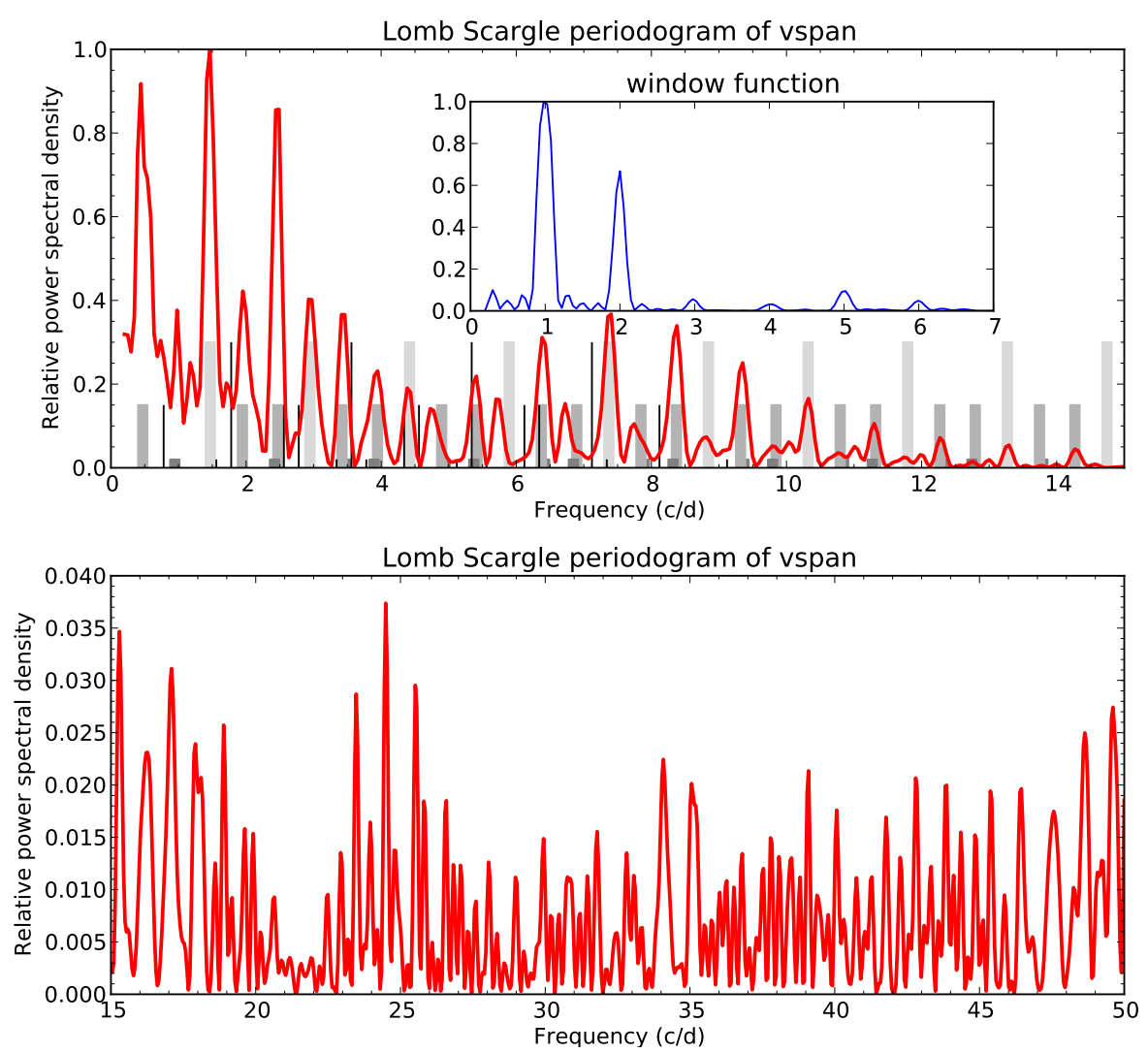

Fig. 5. Lomb Scargle periodogram of vspan, including the window function of the data set. The grey bars at 0.3 height indicate the rotational frequency of the star as determined by Alina et al. (2012), i.e. $1.47 \mathrm{~d}^{-1}$, as well as its harmonics (multiple of this frequency). Its width corresponds to the error bar. The lower bars at 0.15 and 0.02 height indicate the position of the \pm 1 and 2-day aliases with respect to the rotational frequency comb, respectively. The thin vertical black line indicates the same kind of frequency grid for a frequency of $1.77 \mathrm{~d}^{-1}$ (see Sect. 3.3).

each frequency combination, we assumed that we had correctly identified them and calculated the distance to the expected position. The average distance is only $0.043 \mathrm{~d}^{-1}$ and therefore tends to confirm our identification.

A possible coincidence, but nevertheless striking, is that not all harmonics are equally seen in the vspan periodogram: $F_{\text {rot }}$, $2 F_{\text {rot }}, 3 F_{\text {rot }}, 5 F_{\text {rot }}$, and $7 F_{\text {rot }}$ are seen, while $4 F_{\text {rot }}, 6 F_{\text {rot }}$, and $8 F_{\text {rot }}$ are absent. If vspan represents a cleaned radial velocity measurement, concentrating only on the activity within the line and not on its global shift, a simple simulation as shown in Fig. 6 can provide some explanation. Adopting the inclination of Vega's rotation axis with respect to the line of sight $\left(i=7^{\circ}\right)$, i.e. a star seen almost pole-on, we simulated using the basic trigonometric equations the impact of a spot, depending on its latitude, on the stellar radial velocity. We normalized radial velocity and power in the Lomb Scargle periodogram for the spot at $+60^{\circ}$, and omitted any consideration of limb and gravitational darkening, true spot contrast, and extension. The model is purely trigonometrical but shows nicely that spots located close to the pole are seen permanently and provide an almost purely sinusoidal variation, yielding a single peak in the periodogram. Once the spot is at significantly lower latitudes, a first harmonic appears. Owing to the absence of spot structure during half the rotation period, a spot location exactly at the equator will yield cancellation of the $4 F_{\text {rot }}, 6 F_{\text {rot }}, 8 F_{\text {rot }}$ harmonics. Does this indicate the location of potential surface spots close to the equator? At even lower latitudes the spot is only seen for a very short time during the rotation period, therefore yielding all harmonics without cancelling any harmonics.

In the next step, we performed a frequency analysis of the variation in the two different radial velocity estimators. Figure 7 shows the result of a Lomb Scargle periodicity analysis of the variation in both radial velocity estimators with time. The corresponding frequencies are listed in Tables 3 and 4.

As can be seen in Fig. 8, vspan is slightly anticorrelated to the radial velocity (first moment), which reinforces the idea of a starspotted surface. The anticorrelation is more or less pronounced mainly depending on the upper and lower bisector intervals chosen; more precisely, an upper bisector range moving towards higher values generates a stronger anticorrelation in our case. Several tests with different bisector ranges have been performed by also applying the traditional boundary values by Queloz et al. (2001). They yield similar results, and the strong presence of the rotational frequency is obvious in all cases. The method applied for radial velocity determination also reveals some impact on the level of anticorrelation as shown in Fig. 9. Only a very weak effect can be seen as a function of the line selection minimum threshold, a higher threshold yielding a slightly more pronounced anticorrelation.

During our analysis of the data set we did computations with different line masks, and some masks yielded a much more obvious anticorrelation. Since we concentrate on one specific line mask in this article, we prefer showing the corresponding figure.

The rotation period is again well represented in both data sets (F1b, F4c), and many harmonics and respective aliases of the window function are present. Amplitudes are different, which is due to different estimators. In the frequency analysis of the radial velocity (first moment), SigSpec extracts $0.77 \mathrm{~d}^{-1}$ as the highest amplitude frequency. We concluded, however, that Sigspec selected the $-1 \mathrm{~d}$ alias of F1c, which should be F1c $=1.77 \mathrm{~d}^{-1}$ (and probably $F 2 b=1.89 \mathrm{~d}^{-1}$, despite the rather large difference in frequency). We compared two complete frequency grids (fundamental frequency at $0.77 \mathrm{~d}^{-1}$ or $1.77 \mathrm{~cd}$, plus harmonics and window function aliases) with the observed periodogram and based on the frequency distribution, concluded that the true frequency 

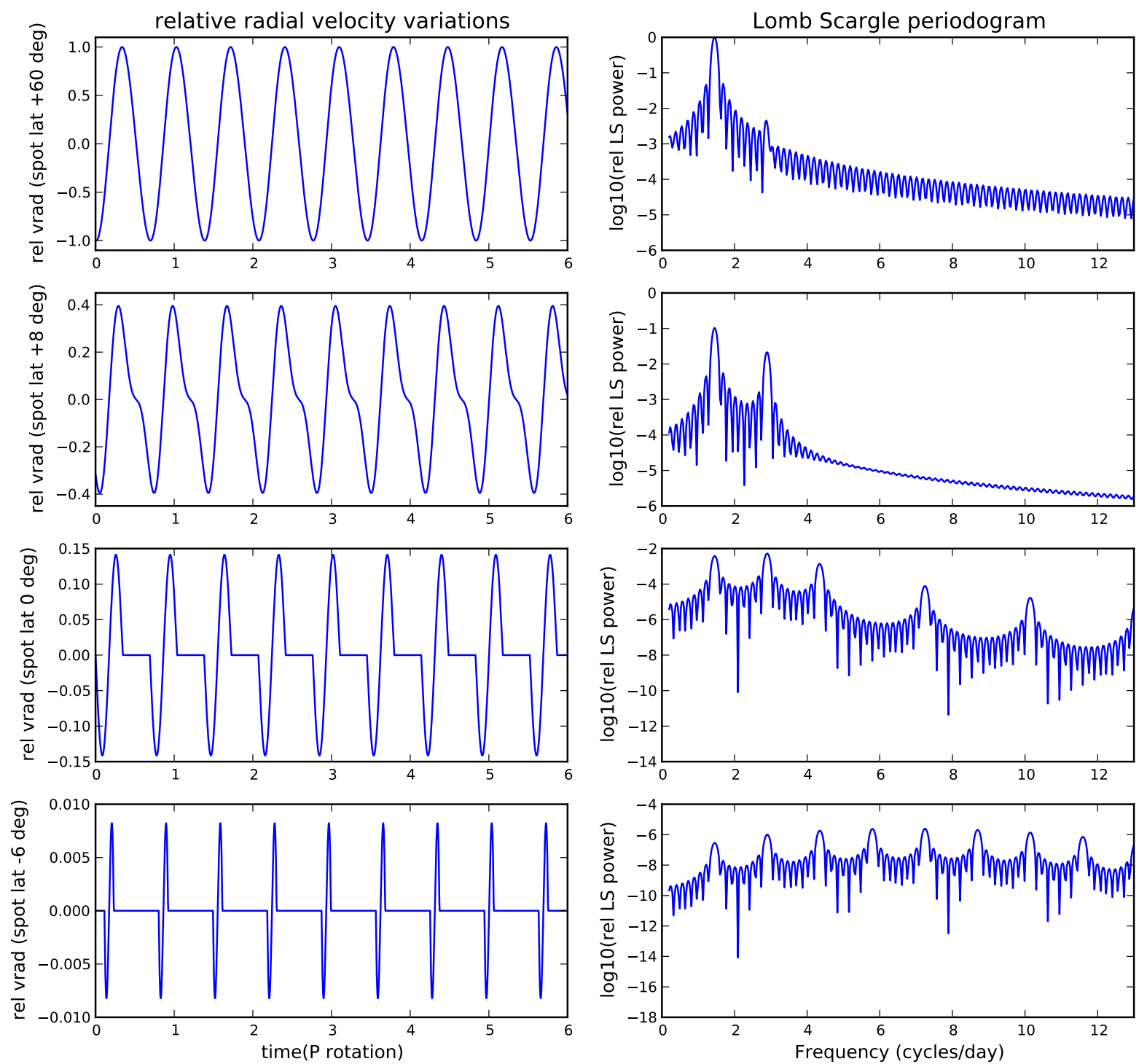

Fig. 6. Simulation of the relative radial velocity variations induced by a spot (of same size) located at latitude (figures left, top to bottom): $+60^{\circ},+8^{\circ}$, $0^{\circ}$ and $-6^{\circ}$ for Vega (inclination angle $i=7^{\circ}$; the star is seen almost pole-on). Figures right, top to bottom: respective Lomb Scargle periodograms. The spot position at latitude $+60^{\circ}$ is taken as the reference with respect to the radial velocity and Lomb Scargle power spectrum amplitude.

must be $\mathrm{F} 1 \mathrm{c}=1.77 \mathrm{~d}^{-1}$ and that the grid for 0.77 is much narrower and provides a significantly less good fit. The discussion of a potential origin of this frequency, which is not detected in vspan, is discussed in Sect. 3.3.

\subsection{Spot-like trails in dynamic spectra}

We propose to further investigate the nature of photospheric features responsible for the $0.678 \mathrm{~d}$ variability showing up in the time series of radial velocities and velocity spans, and gather more evidence of the rotational origin of this periodical signal.

Figure 10 shows a D-shaped bisector that is typical of early type stars (Gray 2010). As can be seen in this figure, enhanced "bisector activity" (as indicated by the spread in the left part of the figure, but also on the standard deviation as represented on the right side) of the bisector variations is seen around the bottom of the profile, but also near continuum. The enhanced variability of the bisector in the lower part is easily understood if surface structures cross the projected stellar disk during rotation. An easy explanation for the upper variations cannot be provided at this stage. Very tiny LSD profile variations were seen in a thorough inspection (Fig. 11).

To highlight the tiny spectral features responsible for the periodic signal, we first cleaned up the data set by getting rid of LSD equivalent photospheric profiles affected by the lowest $\mathrm{S} / \mathrm{N}$, therefore discarding about $1.4 \%$ of all available data. (Files with less than $95 \%$ of the mean $\mathrm{S} / \mathrm{N}$ were rejected.) The next step was to correct the LSD profiles from changes in their equivalent width (EW), observed as a systematic increase in the EW during each observing night. The daily repeatability of this effect suggests an effect linked to the airmass of observations (Fig. 12).

As this smooth evolution is observed to correlate with the $\mathrm{S} / \mathrm{N}$ of LSD profiles, we can remove most of the systematic trend through the assumption that the $\mathrm{EW}$ is a polynomial function of the $\mathrm{S} / \mathrm{N}$. For each night, a second-order polynomial was adjusted to the EW of LSD profiles as a function of the $\mathrm{S} / \mathrm{N}$, and the polynomial trend was then removed (ensuring that the average EW of the final distribution is the same as the initial one). We finally computed an averaged LSD profile for the entire run and subtracted this mean from each LSD profile, to highlight 

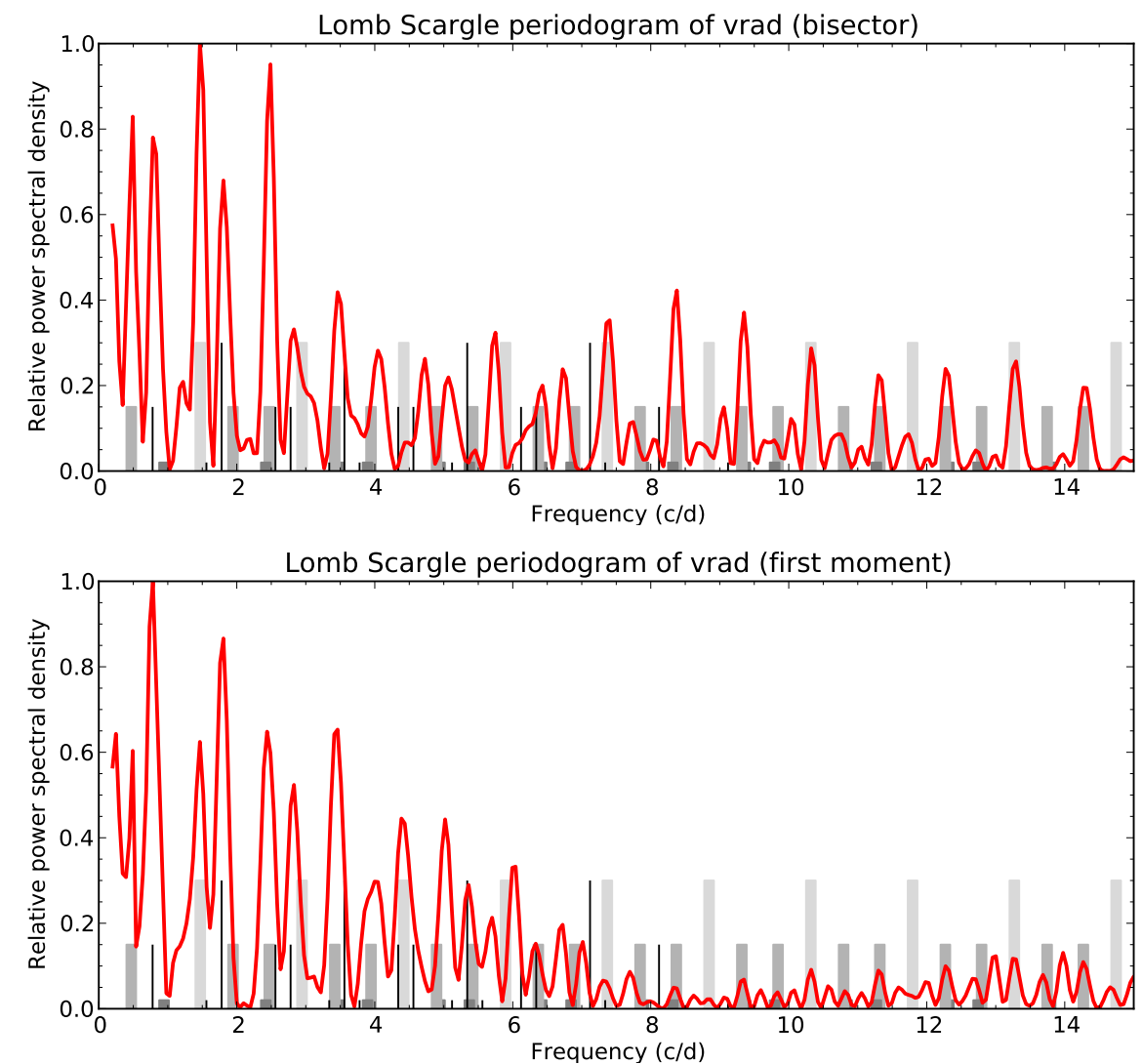

Fig. 7. Lomb Scargle periodogram of the radial velocity evolution during the run, measured by (i) the lower part of the bisector (top) or (ii) the first moment of the profile (bottom). The definition of vertical bars and lines are identical to Fig. 5.

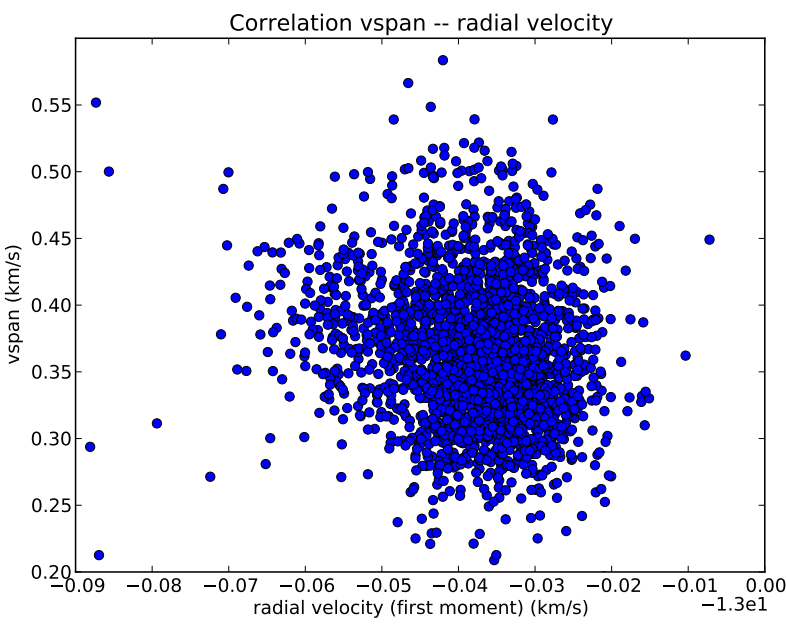

Fig. 8. Anticorrelation of vspan versus vrad (first moment).

fluctuations in the pseudo-line profiles. It should be noted that the periodogram of the EW variations (Fig. 14) does not match any of the other periodograms (except the window function) and that in particular it does not show the rotational frequency or the $1.77 \mathrm{~d}^{-1}$ frequency.

The resulting line residuals are plotted in Fig. 13, after attributing a phase to each observation according to the $0.678 \mathrm{~d}$ period and choosing BJD $=2456142.3308$ (first stellar spectrum of the run) as the phase reference. The dynamic spectra display a number of bright and dark trail-like features first showing up in the blue wing of the line profile, and progressively shifting towards the red wing, in excellent agreement with the typical spectral signature of brightness inhomogeneities carried across the visible stellar hemisphere during stellar rotation, as

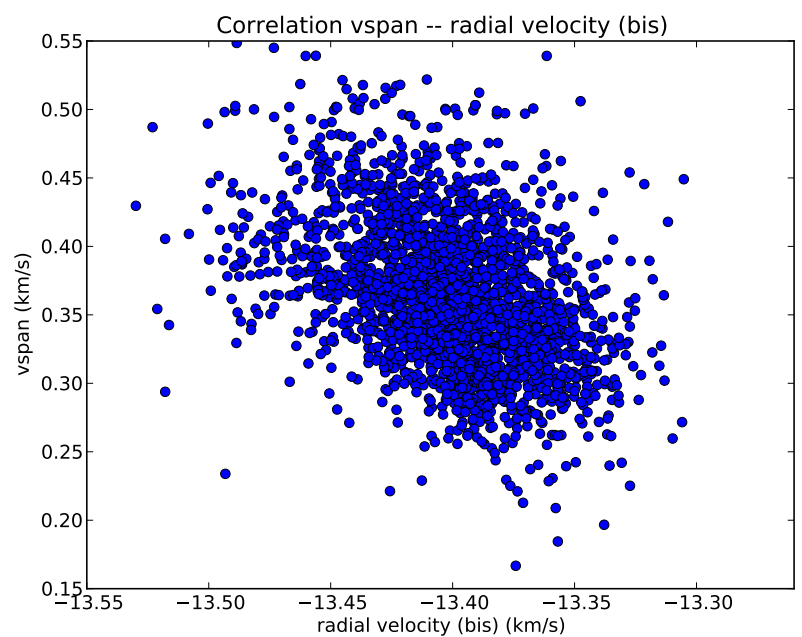

Fig. 9. Anticorrelation of vspan versus vrad (bisector).

routinely observed in active solar-type stars (Collier Cameron et al. 2002). These brightness inhomogeneities are sometimes referred to as "spots" and, if more extended, "plages" in the rest of the present article, regardless of their physical origin and brightness ratio relative to the quiet photosphere (darker or brighter regions). The amplitude of these subtle bumps and dips is of the order of $5 \times 10^{-4}-10^{-3}$ of the continuum. The $2 \mathrm{D}$ phase plots showing the transient spots are conclusive for all line-lists corresponding to different rejection thresholds. However, some artefacts are present if the chosen threshold is too small. (A threshold of 0.1 or 0.2 includes flat-bottomed profiles of weak lines in the line lists, which mixes with the mainly rotational profile of the stronger lines.) 
T. Böhm et al.: Discovery of starspots on Vega
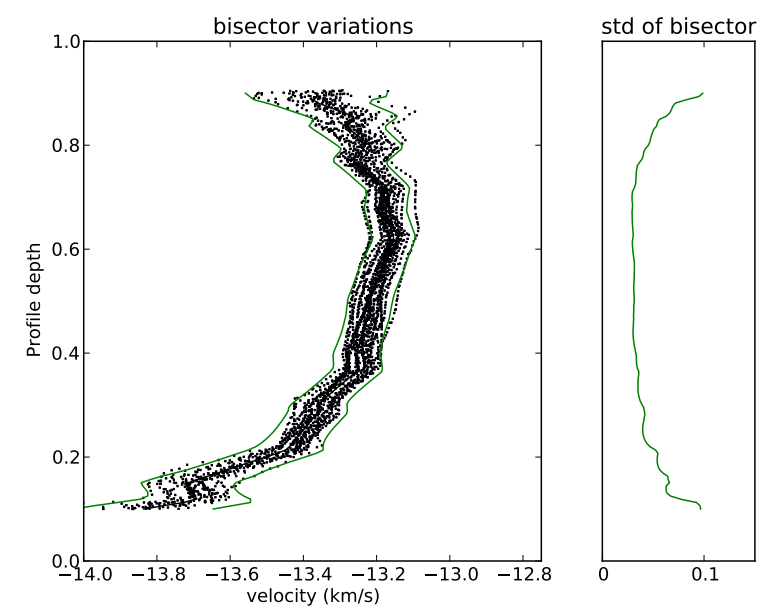

Fig. 10. Distribution of bisectors in the total data set is represented in the left-hand figure. The green lines enclose $95 \%$ credibility intervals for each depth. Right-hand figure: standard deviation of the bisector variations as a function of renormalized profile depth.

The resolution of the spectrograph is $R=75000$, therefore the resolved element corresponds to $4 \mathrm{~km} \mathrm{~s}^{-1}$ in velocity. The typical width of each individual feature on the $2 \mathrm{D}$ phase plots of Fig. 13 are roughly one fifth of the $v \sin i$ or $4.4 \mathrm{~km} \mathrm{~s}^{-1}$ (see for instance trails at phase 0 and velocity 0 ). The trail widths are therefore barely resolved, and we cannot make any conclusion about the minimum spot or plage size on the star's surface. Since the trail width is close to the resolution of the instrument, one could conclude, however, that their size on the stellar surface should be significantly smaller than the angular width corresponding to the trail size broadened by resolution, which is one tenth of $180^{\circ}$. It is therefore likely that sizes of the plages do not exceed $5^{\circ}$ in angular extension.

The main trails are repeatedly observed during different observing nights, demonstrating that they do follow the $0.678 \mathrm{~d}$ period and possess a lifetime of at least a few days. For example, the trail starting around 0 velocity at phase 0.1 can be seen every night at the same phase. The trail pattern (dark-bright-dark) close to $-17 \mathrm{~km} \mathrm{~s}^{-1}$ reappears each night at phase 0.25 . A backtravelling trail can be seen on nights $1-2$ and 3-4 (night 5 did not cover this phase) starting at phase 0.5 and at $+10 \mathrm{~km} \mathrm{~s}^{-1}$ and reaching $-10 \mathrm{~km} \mathrm{~s}^{-1}$ at phase 0.8 . Many more examples can be found be comparing the three plots of Fig. 13. A residual of EW variations is still visible in the dynamic spectra at a level roughly similar to the spot-like trails (and is best observed as a systematically darker line core at the end of each night).

If the trail amplitude is barely above noise level in the bluered transit, the associated red-blue transit (visible in principle for spots that are not eclipsed during stellar rotation thanks to their high latitude) are even fainter, owing to the less favourable projection factor and limb darkening during the return transit. Still, a close look at the left-hand and middle panels of Fig. 13 reveals clearly back-travelling spot signatures.

In addition, a close inspection of individual trails shows further that the trail inclination in the dynamic spectrum is not identical for all spot-like features. As an illustration, the bright trail crossing the line centre each night at phase $\approx 0.2$ looks steeper than the other bright trail crossing line centre at phase $\approx 0.05$. The most natural interpretation of the different trail inclinations is a difference in the stellar latitude of brightness features.

Figure 15 reveals the results of a systematic attempt for optimizing the pre-estimated rotational period of $P=0.678 \mathrm{~d}$. In

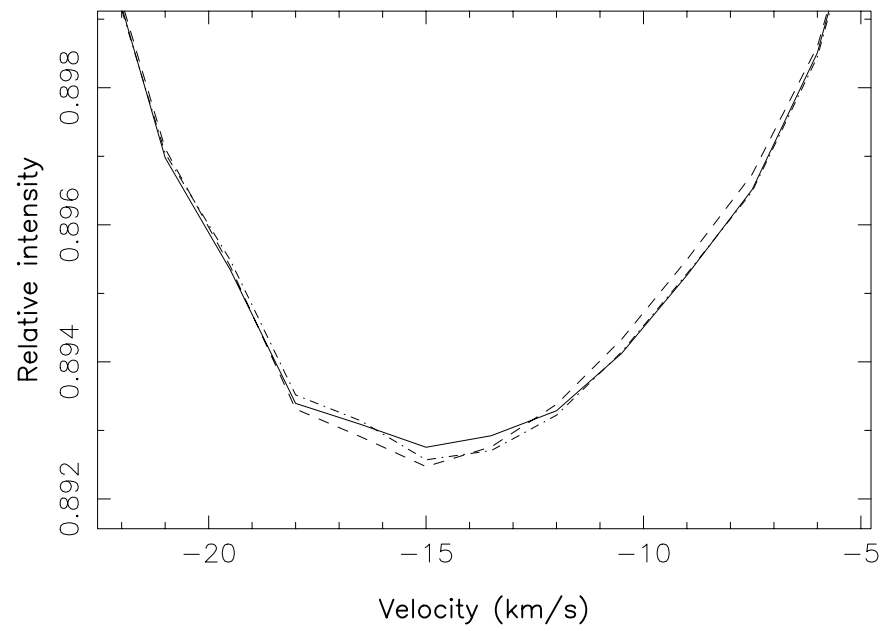

Fig. 11. Variation in the LSD profile, averaged hour per hour, during the first three hours of the first night of the run (Aug. 2, 2012), showing travelling bumps. Continuous line: first hour (20-21:00 UT); dashed line: second hour (21-22:00 UT); dot-dashed line: third hour (22-23:00 UT).

practical, we scanned all periods in a range from 0.55 to $0.80 \mathrm{~d}$, around the period of rotation (as determined by Alina et al. 2012, and this paper). After rephasing all spectra with a given period, we calculated the average standard deviation within a binned subgrid in the velocity or phase space. The lowest consolidated standard deviation occurs when the rephasing period is optimized, i.e. when the residual intensities tend to be similar within the boxes of the subgrid. To assess significance, we did the same analysis on a randomly shuffled data set (randomly associating a time value of the data set to a spectrum). The dotted cloud shows no minimum at all around the stellar rotation period and therefore clearly indicates that the observed minimum of our rephased data set is not due to random fluctuations of noisy data.

From this series of simple observations, we can draw two conclusions: (a) the $0.678 \mathrm{~d}$ period can safely be interpreted as the stellar rotation period; and (b) the spectral rotational modulation is produced by a complex pattern of spots peppering stellar surface. It is tempting to link these intricate brightness inhomogeneities to the complex magnetic field topology previously reported by Petit et al. (2010).

The earlier data sets from 2008, 2009, and 2010 (Böhm et al. 2012 ) were acquired with poorly stabilized spectrographs, and data reduction could correct for these errors only to some extent. Low-frequency information was therefore totally lost, which is the reason we did not directly detect the frequency of stellar rotation in their radial velocity data. Encouraged by our current results, we will try to present, in a forthcoming work, the results of a direct search for rotationally modulated LSD profiles in these data sets, a similar approach to the one presented in this section.

\subsection{Stellar oscillations and a potential exoplanet signature?}

In Böhm et al. (2012), we announced the presence of higher frequencies in the radial velocity periodograms of Vega and suggested the possible detection of corresponding stellar oscillations. We only detected higher frequencies 5.32 and $9.19 \mathrm{~d}^{-1}$ $\left(A \approx 6 \mathrm{~m} \mathrm{~s}^{-1}\right)$ in $2008,12.71$ and $13.25 \mathrm{~d}^{-1}\left(A \approx 8 \mathrm{~m} \mathrm{~s}^{-1}\right)$ in 2009, and 5.42 and $10.82 \mathrm{~d}^{-1}\left(A \approx 3-4 \mathrm{~m} \mathrm{~s}^{-1}\right)$ in 2010. As mentioned in the last section, these data sets were acquired with 


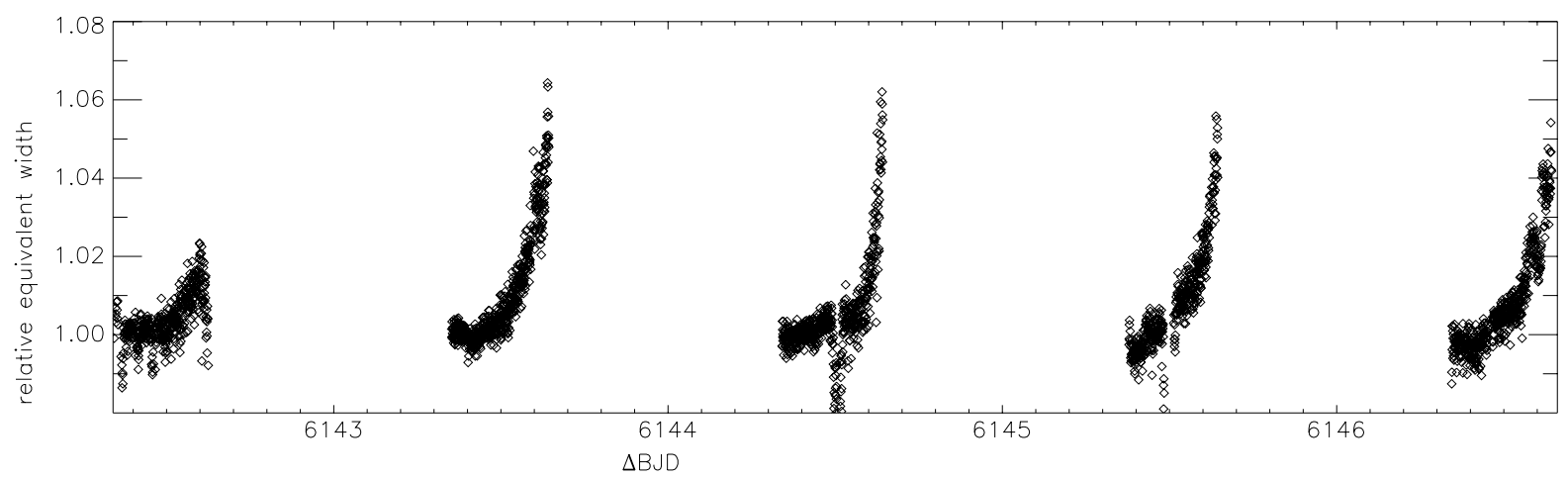

Fig. 12. Nightly relative EW variations of the LSD profiles of this run. Time is expressed in BJD $=2450000+\Delta \mathrm{BJD}$.
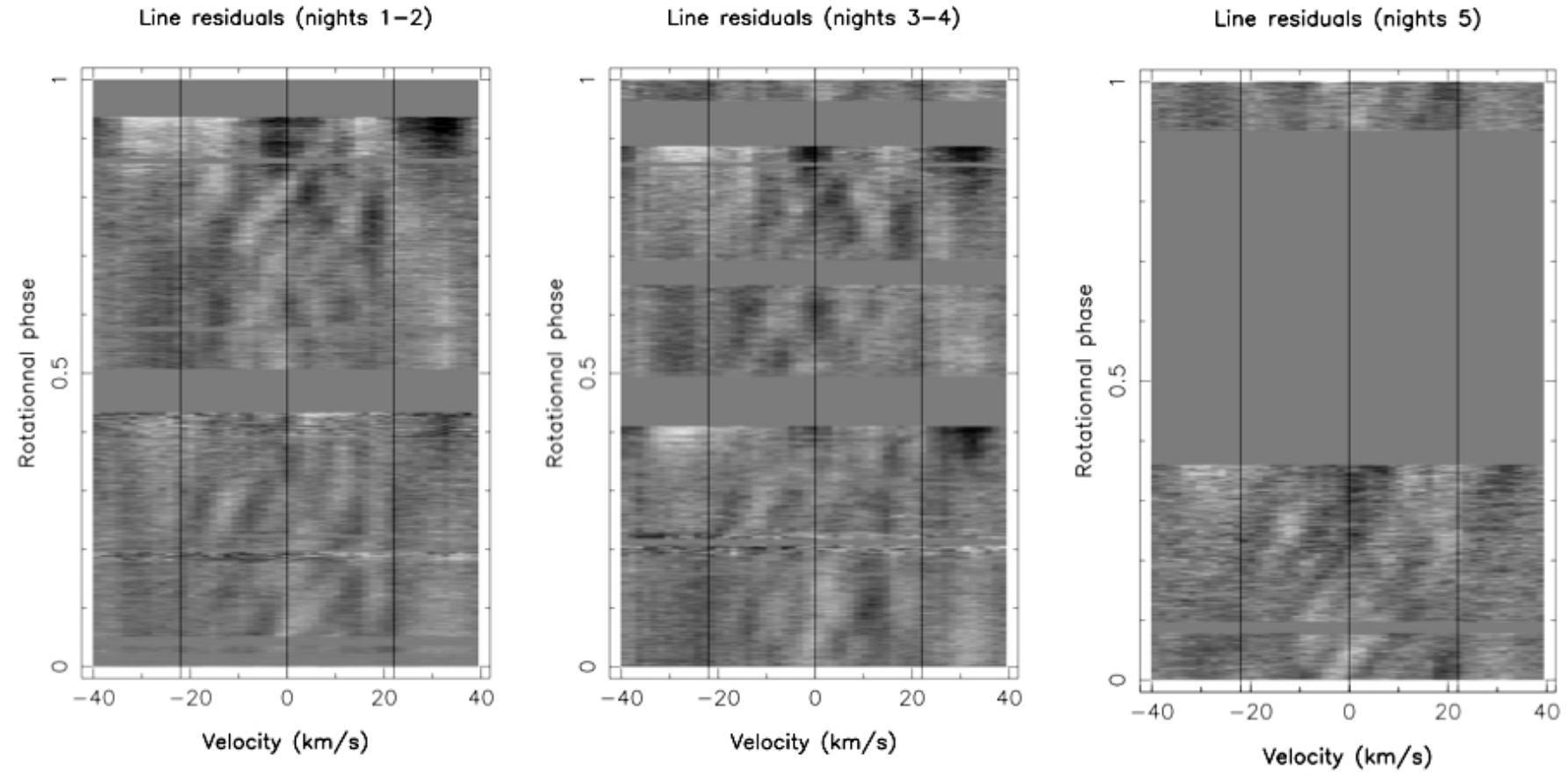

Fig. 13. Residuals after nightly shift correction and subtraction of average profile plotted as a function of velocity and time modulo period, where the period is fixed at the formally estimated value of $P=0.678 \mathrm{~d}$. The coherent structures along the diagonals in this plot are evidence of activity zones (in emission or absorption) moving with stellar rotation and therefore crossing the line profile. The good match of starspot features over several nights is a strong indicator of a structured surface on Vega.

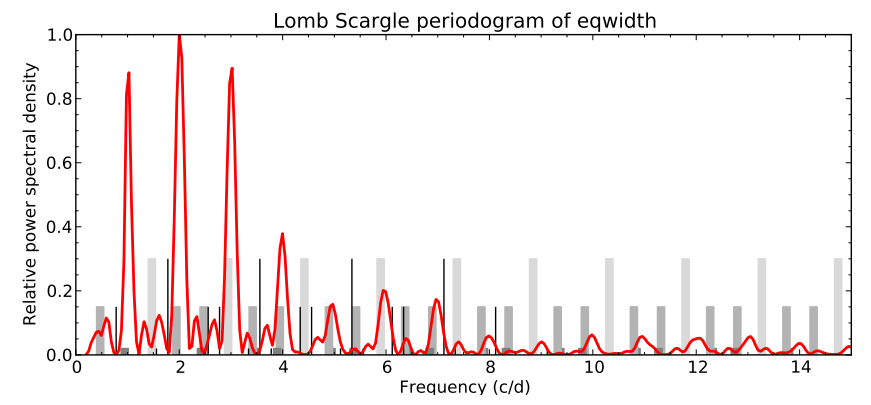

Fig. 14. Lomb Scargle periodogram of the EW variation during the run. Definition of vertical bars and lines are identical to Fig. 5.

instruments that were not optimized in the sense of radial velocity stabilization, making any low-frequency detection, such as stellar rotation, impossible.
As can be seen in Tables 3 and 4 corresponding to our 2012 run, some energy in the periodograms is located in the higher frequency domain. Possible identifications with frequencies found in the former data sets are indicated. Still, the dense frequency spacing of the rotational harmonic comb and its different window function aliases, together with propagated error bars, almost always allow a possible identification to be found with rotationally linked frequencies. This tells us that rotation might be responsible in all data sets for the observed higher frequencies, too. However, it still does not exclude the presence of oscillations in all these data sets, but only prohibits any conclusion at this stage.

The most striking difference between the periodicity analysis of vspan and the two different radial velocity determinations is that the strong frequency at $1.77 \mathrm{~d}^{-1}$ (or $1.89 \mathrm{~d}^{-1}$ ) only appears in the latter ones (see Fig. 16). For the sake of completeness, we want to mention that these additional frequencies do not appear when selecting only the strongest lines (threshold of 0.7 ). In that case the number of lines drastically diminishes to only 


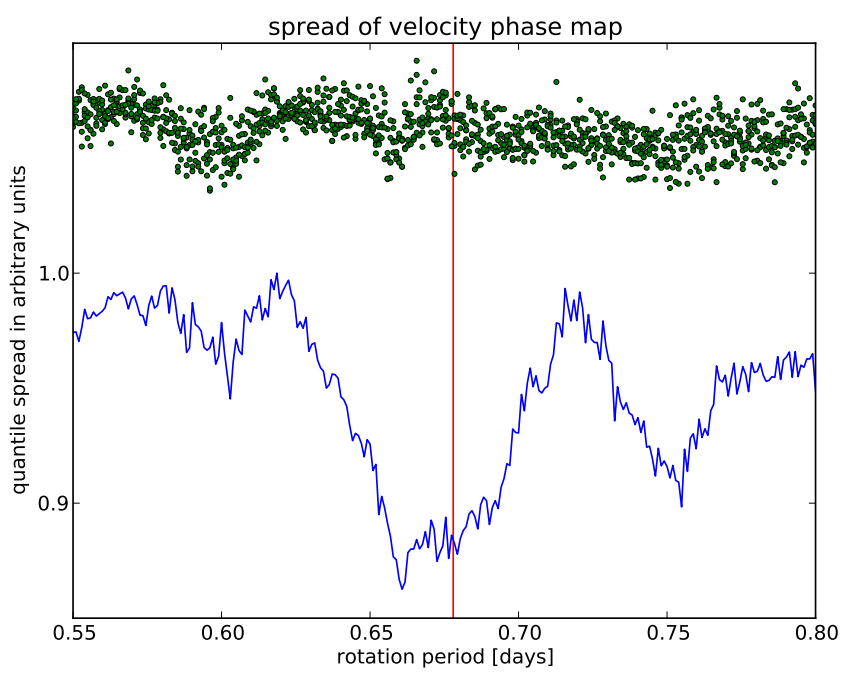

Fig. 15. Optimal coherence is searched for a temporal remapping of the data set presented in Fig. 13 around the pre-estimated rotational period. Optimality is based on the spread of the cloud in three dimensions (binned data in time modulo Prot, intensity, velocity). It can be seen that Prot $=0.678 \mathrm{~d}$ corresponds to the best matching period (blue line). To assess significance we used the same data set with a randomly permutated time data set (green dots).

144 lines, and all periodograms become messy, with no clear structure. For thresholds of 0.1 and 0.3 , the number of lines used for the LSD correspond to 1494 and 296, respectively, yielding a much higher $\mathrm{S} / \mathrm{N}$ equivalent photospheric profile. Vspan measures an asymmetry within the profile and is totally insensitive to general radial velocity shifts. Should we therefore understand $\mathrm{F} 1 \mathrm{c}=1.77 \mathrm{~d}^{-1}$ (or F2b) as the signature of a general, i.e. linked to orbital dynamics, shift in the line profile?

The presence of an exoplanet could be one possible source of such bulk radial velocity variations. Activity-induced radial velocity signature (starspots or bumps travelling through the line profile) could not provide this type of overall variation. In Fig. 7 one can see that F1c is most likely in the presence of two harmonics at 2 F1c and 3 F1c. A low-excentricity orbit could explain a low number of harmonics. Since amplitudes differ significantly between both radial velocity measurements, only very gross deductions can be attempted. Applying Kepler's laws and Vega's fundamental parameters, using the overall shift as the radial velocity (determined by the first moment), and supposing an exoplanet orbiting in the equatorial plane at a distance of $0.017 \mathrm{AU}$, a $0.34 M_{\text {Jupiter }}$ exoplanet could satisfy the observed parameters (stellar mass: $2.15 M_{\odot}$, amplitude $v_{\text {orb(Vega) }} \sin i \approx$ $6 \mathrm{~m} \mathrm{~s}^{-1}$ derived from the first moment measurement, inclination angle of the system: $7^{\circ}$ (pole-on), $P=0.56 \mathrm{~d}$ (corresponding to $\left.\mathrm{F} 1 \mathrm{c}=1.77 \mathrm{~d}^{-1}\right)$, and eccentricity e $=0$ ). Using the values of vrad (bisector), i.e. $P=0.53 \mathrm{~d}$ and $v_{\text {orb(Vega) }} \sin i \approx 22.9 \mathrm{~m} \mathrm{~s}^{-1}$ an exoplanet mass of $1.24 M_{\text {Jupiter }}$ at 0.0165 AU would satisfy the equations. In both case studies, the interesting result is the proximity of the frequency to the rotation frequency. It indicates that the orbital radius of such an exoplanet would correspond to only 1.36 (or 1.31) $R_{\text {Vega }}$ (calculated for $e=0$ and using the concordance model as published by Monnier et al. 2012), while the co-rotating radius or synchronous orbit corresponds to $1.5 R_{\text {Vega }}$.

If at this stage we have no further indication of the potential presence of an exoplanet and if we know that until now only a few exoplanets with such short orbital periods have been detected, attention should be given to the recent results by Balona (2014), who announces that approximately $19 \%$ of A-type stars were potentially accompanied by a roughly Jupiter-mass exoplanet in a synchronous orbit. The most interesting conclusion of our (still hypothetical) analysis is therefore that planetary material with a significant fraction of a Jupiter mass could be located close to the synchronous (or co-rotation) radius. This result seems to agree with the findings of Balona (2014), and Vega could be one of these close-planet A-type stars. If confirmed, it would be very interesting to observe associated tidal effects of such a close planet orbiting a "hot" star. Would these findings indicate that in A-type stars exoplanet migration stops at the corotating radius? Would that imply that dipolar magnetic fields dominate regions out to the corotation radius and suppress any further migration?

\section{Conclusion}

The discovery of corotating structures at the surface of a nonchemically peculiar A-type star provides new insight into the processes at work in the envelope of a typical intermediatemass star. Long-lived large scale chemical spots are already known to exist on $\mathrm{Ap} / \mathrm{Bp}$ stars, but this class of magnetic and chemically peculiar stars only represents $5-10 \%$ of stars in this mass range and the origin of their spots is attributed to the atomic diffusion of chemical elements in an atmosphere stabilized and structured by a strong 300 Gauss or higher large scale fields. Chemical anomalies associated with abundance spots are also seen in HgMn stars, another class of chemically peculiar stars with late-B spectral types (e.g., Makaganiuk et al. 2011; Korhonen et al. 2013). No evidence of magnetic fields has stood up to scrutiny, and upper limits as low as a few $G$ exist for some stars (Kochukhov et al. 2013). These stars show slow rotation rates for hot stars, a property that most likely helps in creating a stable atmosphere where atomic diffusion can operate. In addition to the rotation rate, there is a very significant difference between Vega and these late B-type stars, namely that the late B stars have undetectable micro turbulence, which indicates that they have stable atmospheres. In contrast, Vega has non-zero micro turbulence, which is a property consistent with the abrupt change in micro turbulence velocity at roughly the A0-B9 boundary (Landstreet 1998).

In a non-chemically peculiar A-type star like Vega, the rotational modulation must have a different origin since with a very low magnetic field and a high rotation rate, the atmosphere cannot be stable enough to generate chemical anomalies through atomic diffusion. Nevertheless, the magnetic field of Vega is still a natural explanation for corotating structures seen with Doppler imaging. In this case, the property of these structures should help determine the enigmatic nature of Vega's magnetic field.

Although the envelope of A-type stars is mainly radiative, small convective layers due to the hydrogen and to the first and the second helium ionizations are present. These layers can in principle host a dynamo driven by the convective motions. Owing to their small thickness and low density, the energy contained in these motions is limited. Moreover, close to the surface, the convective turnover time can be much shorter than the rotation rate, in which case the dynamo is inefficient. Nevertheless, Cantiello \& Braithwaite (2011) find that in the hotter O and B stars, the convective layer due to the opacity peak related with iron group elements can generate a relatively strong magnetic field, assuming equipartition between kinetic and magnetic energy and estimating the convective velocity from the mixing length model. In A-type stars, the convective layer induced by the second helium ionization (Weiss \& Kupka 1999) might play a similar role. However, whether such a dynamo can generate 


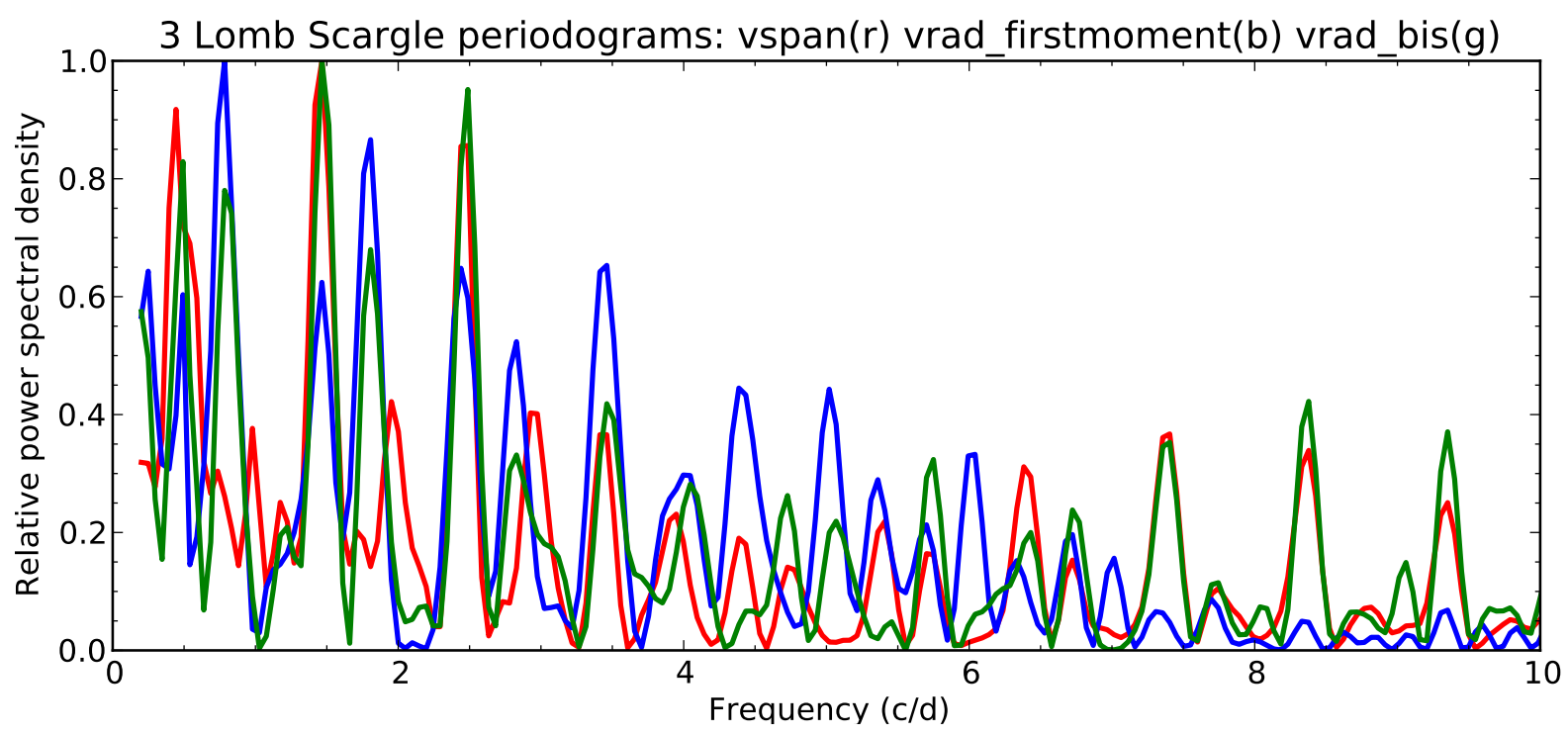

Fig. 16. Lomb Scargle periodogram showing the frequencies present in vspan (red), vrad (first moment, blue), and vrad (bisector, green). Colour codes are online.

the observed 7 gauss, nearly polar spot of Vega (Petit et al. 2014) and also account for the observed co-rotating structures remains to be verified. Furthermore, convective dynamos are intrinsically variable with a spot lifetime close to the rotation period. This variability is not detected in our data.

Other possible origins of Vega's magnetic field, discussed by Lignières et al. (2009) and Braithwaite \& Cantiello (2013), rather involve fields generated in the early phase of the star life and their subsequent evolution in the radiative enveloppe. In this context also, a key feature that would help distinguish between models is the intrinsic time variation of the field. Given the low amplitude of the Stokes $V$ profile, we expect that, if present, this variability will be easier to detect through spectroscopic studies similar to this one.

Another question raised by our study concerns the sign and the amplitude of the luminosity contrast induced by the corotating structure. For the weak magnetic field observed in Vega, bright rather than dark spots are expected because dark spots only occur when the field is strong enough to limit convective heat transport within the spot. As mentioned before, rotational modulations compatible with spots have been detected with Kepler's light curves in a large fraction of A-type stars (Balona 2011). If these modulations are the photometric counterpart of the present spectroscopic structures, this would strongly support the existence of a widespread Vega-like magnetism and activity among A-type stars.

Acknowledgements. The author wants to thank the staff of SOPHIE/OHP for their efficient support. He also acknowledges support from the French national programme PNPS/INSU. M.R. acknowledges financial support from the FP7 project SPACEINN: Exploitation of Space Data for Innovative Helio- and Asteroseismology. GAW is supported by a Discovery Grant from the Natural Science and Engineering Research Council (NSERC) of Canada. The authors thank the ANR Imagine for its support.

\section{References}

Alina, D., Petit, P., Lignières, F., et al. 2012, in AIP Conf. Ser. 1429, eds. J. L. Hoffman, J. Bjorkman, \& B. Whitney, 82

Antonello, E., Mantegazza, L., Rainer, M., \& Miglio, A. 2006, A\&A, 445, L15 Aufdenberg, J. P., Mérand, A., Coudé du Foresto, V., et al. 2006, ApJ, 645, 664
Aurière, M., Donati, J.-F., Konstantinova-Antova, R., et al. 2010, A\&A, 516, L2

Aurière, M., Wade, G. A., Konstantinova-Antova, R., et al. 2009, A\&A, 504, 231 Balona, L. A. 2011, MNRAS, 415, 1691

Balona, L. A. 2013, MNRAS, 431, 2240

Balona, L. A. 2014, MNRAS, 441, 3543

Bazot, M., Ireland, M. J., Huber, D., et al. 2011, A\&A, 526, L4

Böhm, T., Lignières, F., Wade, G., et al. 2012, A\&A, 537, A90

Braithwaite, J., \& Cantiello, M. 2013, MNRAS, 428, 2789

Budkovskaya, V. 2013, Izvestiya Krymskoi Astrofizicheskoi Observatorii, 109, 132

Cantiello, M., \& Braithwaite, J. 2011, A\&A, 534, A140

Collier Cameron, A., Donati, J.-F., \& Semel, M. 2002, MNRAS, 330, 699

Donati, J.-F., Semel, M., Carter, B. D., Rees, D. E., \& Collier Cameron, A. 1997, MNRAS, 291, 658

Dumusque, X., Pepe, F., Lovis, C., et al. 2012, Nature, 491, 207

Gray, D. F. 2010, ApJ, 721, 670

Gray, R. O. 1985, JRASC, 79, 237

Gray, R. O. 2007, in The Future of Photometric, Spectrophotometric and Polarimetric Standardization, ed. C. Sterken, ASP Conf. Ser., 364, 305 Gulliver, A. F., Hill, G., \& Adelman, S. J. 1994, ApJ, 429, L81

Hill, G., Gulliver, A. F., \& Adelman, S. J. 2004, in The A-Star Puzzle, eds. J. Zverko, J. Ziznovsky, S. J. Adelman, \& W. W. Weiss, IAU Symp., 224, 35 Hill, G., Gulliver, A. F., \& Adelman, S. J. 2010, ApJ, 712, 250

Kochukhov, O., Makaganiuk, V., \& Piskunov, N. 2010, A\&A, 524, A5 Kochukhov, O., Makaganiuk, V., Piskunov, N., et al. 2013, A\&A, 554, A61 Korhonen, H., González, J. F., Briquet, M., et al. 2013, A\&A, 553, A27 Kurtz, D. W., \& Müller, M. 1999, MNRAS, 310, 1071 Landstreet, J. D. 1998, A\&A, 338, 1041

Lignières, F., Petit, P., Böhm, T., \& Aurière, M. 2009, A\&A, 500, L41 Makaganiuk, V., Kochukhov, O., Piskunov, N., et al. 2011, A\&A, 529, A160 Mantegazza, L., Poretti, E., Michel, E., et al. 2012, A\&A, 542, A24

Monnier, J. D., Che, X., Zhao, M., et al. 2012, ApJ, 761, L3

Morgenthaler, A., Petit, P., Saar, S., et al. 2012, A\&A, 540, A138 Nardetto, N., Poretti, E., Rainer, M., et al. 2014, A\&A, 561, A151

Peterson, D. M., Hummel, C. A., Pauls, T. A., et al. 2006, Nature, 440, 896 Petit, P., Donati, J.-F., Aurière, M., et al. 2005, MNRAS, 361, 837 Petit, P., Lignières, F., Wade, G. A., et al. 2010, A\&A, 523, A41

Petit, P., Lignières, F., Wade, G. A., et al. 2014, A\&A, 568, C2 Queloz, D., Henry, G. W., Sivan, J. P., et al. 2001, A\&A, 379, 279 Reegen, P. 2007, A\&A, 467, 1353

Reiners, A., \& Schmitt, J. H. M. M. 2003, A\&A, 398, 647

Takeda, Y., Kawanomoto, S., \& Ohishi, N. 2008, ApJ, 678, 446

Uytterhoeven, K., Mathias, P., Poretti, E., et al. 2008, A\&A, 489, 1213

Weiss, W. W., \& Kupka, F. 1999, in Stellar Structure: Theory and Test of Connective Energy Transport, eds. A. Gimenez, E. F. Guinan, \& B. Montesinos, ASP Conf. Ser., 173, 21

Yoon, J., Peterson, D. M., Kurucz, R. L., \& Zagarello, R. J. 2010, ApJ, 708, 71 